\title{
Comparação antropométrica e do perfil glicêmico de idosos diabéticos praticantes de atividade física regular e não praticantes
}

\section{Anthropometric comparison and the glycemic profile in elderly diabetics practitioners and non- practitioners of regular physical activity}

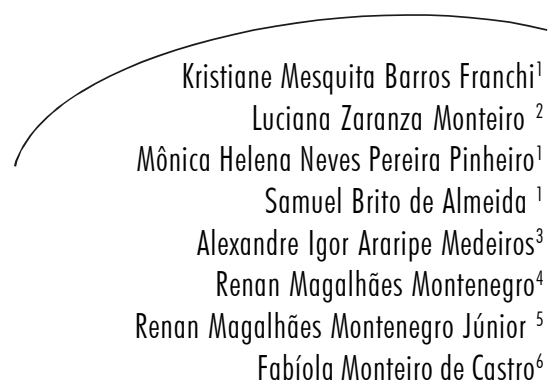

O objetivo do estudo foi comparar os parâmetros antropométricos e perfil glicêmico de idosos diabéticos tipo 2 praticantes de atividade física regular e não praticantes. Métodos: $\mathrm{O}$ estudo, do tipo transversal, envolveu 70 idosos com diabetes mellitus tipo 2, com idade entre 60 e 80 anos. A coleta de dados foi através de questionário estruturado abordando: a) características demográficas dos pacientes (idade e nível de escolaridade) e b) características do padrão de atividade física (frequência, duração (min) e tempo (meses) da prática de exercícios físicos). Foram realizadas medidas antropométricas e verificação do perfil glicêmico (glico-hemoglobina). Os dados foram analisados no programa STATA versão 9.0. Foram calculados a média e desvio padrão da média (DP) e realizado o teste de análise de variância (ANOVA). Para verificar a associação de variáveis qualitativas, utilizou-se o teste exato de Fisher e as associações entre as variáveis quantitativas foram estimadas através do coeficiente de correlação de Pearson, com uma confiança de 95\%. O nível de significância foi de $\mathrm{p}<0,05$. Resultados: Em relação ao nível de escolaridade, não houve influência na realização da prática de atividade física. Nos PAF observa-se uma diminuição do IMC e do perfil glicêmico, quando comparados aos NPAF. A caminhada foi a atividade física mais realizada pelos idosos (51,5\%). A partir dos resultados obtidos na análise dos dados, foi possível mostrar os benefícios que a atividade física proporciona aos indivíduos no processo de envelhecimento e no bom controle glicêmico.

\footnotetext{
Universidade de Fortaleza. Departamento de Educação Física. Fortaleza, CE, Brasil

2 Universidade de São Paulo. Faculdade de Medicina de Ribeirão Preto, Departamento de Clínica Médica - Endocrinologia. Ribeirão Preto, SP, Brasil

3 Universidade do Porto. Pós-graduação em Treino de Alto Rendimento Desportivo. Porto, Portugal.

4 Universidade Federal do Ceará. Faculdade de Medicina, Departamento de Educação Física. Fortaleza, CE, Brasil

5 Universidade Federal do Ceará. Faculdade de Medicina, Departamento de Saúde Comunitária. Fortaleza, CE, Brasil

6 Universidade Federal do Ceará. Faculdade de Medicina, Serviço de Endocrinologia e Diabetes do Hospital Universitário Walter Cantídio. Fortaleza, CE, Brasil
}

Palavras-chave: Idoso. Diabetes Mellitus Tipo 2. Antropometria. Índice Glicêmico. Perfil de Saúde. Atividade Motora. Estilo de Vida Sedentário. Estudo Comparativo. Análise Transversal. Atividade Física 


\section{Abstract}

This study aimed to compare the anthropometric parameters and plasma glucose in elderly with type 2 diabetes who practice regular physical activity or not. Methods: The cross-sectional study comprised 70 elderly people with type 2 diabetes mellitus (DM2), aged between 60 and 80 years. Data collection was through a structured questionnaire: a) demographic characteristics of patients (age and level of schooling) and b) characteristics of the pattern of physical activity (frequency, duration (min) and time (months) the practice of physical exercise). Anthropometric measurements were taken and verification of plasma glucose (glyco-hemoglobin). The data were analyzed in the program STATA version 9.0. The data was analyzed using mean and standard deviation of the mean (SD), and analysis of variance (ANOVA); to determine the combination of qualitative variables, it was used the Fisher exact test and associations between quantitative variables were estimated by the coefficient of Pearson's correlation, with $95 \%$ confidence. The level of significance was $\mathrm{p}<0.05$. Results: Concerning education level, there was no influence on the attainment of physical activity. In PAF there is a decrease of IMC and plasma glucose as compared to NPAF. The walk was the most physical activity undertaken by the elderly $(51.5 \%)$. The results obtained in the analysis of data made it possible to show the benefits that physical activity gives individuals in the aging process and in good glycemic control.
Key words: Aged.

Diabetes Mellitus, Type 2. Anthropometry. Glycemic Index. Health Profile. Motor Activity. Sedentary Lifestyle. Comparative Study. Period Analysis. Physical Activity.

\section{INTRODUÇÃOO}

A população brasileira vem envelhecendo de forma rápida desde o início da década de $1960^{1}$ e, de acordo com as projeções estatísticas da Organização Mundial de Saúde (OMS), no ano de 2025 o Brasil será o sexto país do mundo com o maior número de pessoas idosas..$^{2-5}$

Segundo Alves \& Veras, ${ }^{6}$ neste período a população brasileira como um todo estará crescendo cinco vezes mais, enquanto o grupo etário de idade superior a 60 anos estará se ampliando 15 vezes.

O processo de envelhecimento físico, mental e social acarreta alterações corporais que são importantes de serem avaliadas em um plano nutricional. ${ }^{2,3}$

De acordo com o Censo Populacional de 2000, os brasileiros com 60 anos ou mais já somam 14.536.029 indivíduos, representando $8,6 \%$ da população total. ${ }^{7}$ À medida que aumenta a idade cronológica, as pessoas se tornam menos ativas, as capacidades físicas diminuem e, com as alterações psicológicas que acompanham a idade (sentimento de velhice, estresse, depressão), existe ainda diminuição maior da atividade física, que consequentemente facilita a aparição de doenças crônicas, contribuindo para deteriorar o processo de envelhecimento. ${ }^{8}$ A incidência dessas doenças é alta nos indivíduos idosos e o risco de desenvolvêlos ou de torná-las mais graves, levando a incapacidades, deve ser identificado precocemente.

Uma das doenças crônicas que está aumentando é o diabetes do tipo 2, sendo um fator de risco maior para morte e várias complicações não fatais que acarretarão um grande impacto aos pacientes, seus familiares e sistemas de saúde. Estudos têm demonstrado claramente que a doença pode ser efetivamente prevenida por modificações no estilo de vida. ${ }^{?}$

Em verdade, poder-se-ia considerar que o diabetes se tornou uma epidemia nas últimas décadas, devido não só ao avanço na idade das populações, mas também a um substancial aumento na prevalência de obesidade e à diminuição nos níveis de atividade física, ambos passíveis de serem atribuídos ao estilo de vida ocidental. ${ }^{10}$ 
No Brasil, a prevalência de diabetes em indivíduos entre 30 e 75 anos de idade foi estimada em $4,2 \%$, sendo que aproximadamente $70 \%$ estavam em uso de hipoglicemiantes orais ou insulina. ${ }^{11}$

Sedentários apresentam maior ocorrência de diabetes e obesidade do que indivíduos que fazem atividade física. ${ }^{12}$ Esta última se associa significativamente com a redução da prevalência de diabetes tipo 2 e suas morbidades associadas. ${ }^{13,14}$

A atividade física exerce efeito benéfico na tolerância à glicose. A caminhada parece ser um modo conveniente de atividade física de baixo impacto, sendo a mais recomendada para diabéticos. ${ }^{15}$ No entanto, várias formas de atividade física, contanto que praticadas com regularidade e persistência, aumentam o fluxo sanguíneo cutâneo, permitindo a prevenção e a reversão de transtornos vasculares que resultam em úlceras e outras complicações no paciente diabético. ${ }^{16}$

Nesse contexto, este estudo buscou comparar os parâmetros antropométricos e perfil glicêmico de idosos diabéticos tipo 2 praticantes de atividade física regular (PAF) e não praticantes de atividade física (NPAF).

\section{METODOLOGIA}

Estudo descritivo, transversal e quantitativo, realizado com idosos diabéticos residentes na cidade de Fortaleza, CE. A população desta pesquisa foi composta por idosos voluntários, física e mentalmente independentes, entre $60 \mathrm{e}$ 80 anos de idade, portadores de DM2, de ambos os sexos, atendidos no Serviço de Endocrinologia e Diabetes, da Universidade Federal do Ceará (SED-UFC).

Foram incluídos no estudo idosos sem comprometimento de memória que prejudicasse as informações a serem investigadas, com participação voluntária e com assinatura ou impressão digital em Termo de Consentimento Livre e Esclarecido, após orientação quanto aos objetivos e procedimentos da pesquisa. A amostra foi composta por 70 idosos, sendo 35 praticantes de atividade física regular e 35 não praticantes de atividade física.

Para realizar a coleta de dados, utilizaram-se os seguintes protocolos: medidas antropométricas e verificação do perfil glicêmico (glico-hemoglobina). Os parâmetros antropométricos avaliados foram: peso, estatura e Índice de Massa Corporal.

Peso $(\mathrm{Kg})$ - Para efetuar a pesagem, foi utilizada uma balança digital portátil Tec 130 da marca Tech Line ${ }^{\circledR}$ Brasil. Procedimento: o avaliado ficava em pé e descalço com afastamento lateral dos pés, ereto com o olhar fixo à frente. Verificouse e anotou-se o valor dado em $\mathrm{Kg}$ com aproximação de $0,1 \mathrm{Kg}$.

Estatura $(\mathrm{cm})$ - Material: para a mensuração da estatura foi utilizada uma fita métrica graduada em centímetros e décimo de centímetros fixada à parede e um cursor. Procedimento: o avaliado permanecia na posição anatômica, com os pés unidos, colocando em contato com o instrumento de medida as superfícies posteriores do calcanhar, cintura pélvica, cintura escapular e região occipital. A medida foi realizada com o indivíduo em apneia inspiratória e com a cabeça paralela ao solo, feita com o cursor em ângulo de $90^{\circ}$ em relação à escala. O avaliado foi orientado para não se encolher no momento em que o cursor lhe tocasse a cabeça.

Índice de Massa Corporal (IMC) - O IMC determina a relação do peso corporal com a estatura do indivíduo. É calculado através da razão entre o peso (em kg) e a estatura ao quadrado (em metros).

Para a verificação do perfil glicêmico, foi realizada uma medida da glico-hemoglobina $\left(\mathrm{Hb}_{\mathrm{Alc}}\right)$, de amostra sanguínea coletada em um período não superior a dois meses do momento da avaliação e uma medida de glicemia capilar em jejum. A determinação da glico-hemoglobina $\left(\mathrm{Hb}_{\mathrm{A1c}}\right)$ foi realizada no Laboratório Central, do SED-UFC, num mesmo ensaio, utilizando-se Kit da In Vitro Diagnóstica Ltda. De acordo com a Sociedade Brasileira de Diabetes ${ }^{17}$, os valores de referência de normalidade são $\leq 7 \%$. 
Após as avaliações antropométricas e glicêmicas, os participantes responderam a um questionário estruturado abordando: a) características demográficas dos pacientes (idade e nível de escolaridade); e b) características do padrão de atividade física (frequência, duração (min) e tempo (meses) da prática de exercícios físicos).

Os questionários foram aplicados em forma de entrevista por uma equipe previamente treinada, composta por entrevistadores com nível de escolaridade superior e com experiência prévia de trabalho de campo. A coleta de dados foi realizada no momento em que os pacientes esperavam para serem atendidos pelo médico responsável no HUWC-FAMED-UFC.

Os dados foram processados no programa STATA versão 9.0, $2003^{18}$. Foram calculados a média e desvio padrão da média (DP) e realizado o teste de análise de variância (ANOVA). Para verificar a associação de variáveis qualitativas, utilizou-se o teste exato de Fisher e as associações entre as variáveis quantitativas foram estimadas através do coeficiente de correlação de Pearson, com uma confiança de $95 \%$. O nível de significância foi de $\mathrm{p}<0,05$.
Foram respeitados os princípios éticos e legais, de acordo com as recomendações da Resolução CONEP n. ${ }^{\circ} 196 / 96^{19}$. O projeto foi submetido e aprovado pelo Comitê de Ética da Universidade de Fortaleza - UNIFOR (Parecer no 129/2004).

\section{RESULTADOS}

Foram avaliados 70 idosos com DM2, dos quais $68,6 \%(\mathrm{~N}=48)$ eram do sexo feminino e $31,4 \%(\mathrm{~N}=22)$ do sexo masculino. Embora não haja diferença de gênero em termos de prevalência do diabetes, a amostra avaliada teve a predominância de idosos do sexo feminino, o que pode refletir a maior longevidade das mulheres em relação aos homens. Segundo Coelho Filho, ${ }^{20}$ este fenômeno se atribui à menor exposição a determinados fatores de risco, principalmente no trabalho, menor prevalência de tabagismo e uso de álcool, diferenças quanto à atitude em relação a doenças e incapacidades e maior frequência do sexo feminino em atendimentos de saúde.

$\mathrm{Na}$ tabela 1, observa-se que o nível de escolaridade não influenciou na realização da prática de atividade física.

Tabela 1 - Frequência (\% linha) e resultado do teste exato de Fisher para as variáveis "Prática de atividade Física" e "Escolaridade". Fortaleza, CE. 2008.

\begin{tabular}{|c|c|c|c|c|}
\hline \multirow[b]{2}{*}{ Escolaridade } & \multicolumn{2}{|c|}{ Prática de Atividade Física } & \multirow[b]{2}{*}{ Total } & \multirow[b]{2}{*}{$\mathrm{p}$-valor } \\
\hline & Não & $\operatorname{Sim}$ & & \\
\hline \multirow[t]{2}{*}{ Analfabeto } & 8 & 7 & 15 & 0,85 \\
\hline & $53 \%$ & $47 \%$ & $100 \%$ & \\
\hline \multirow[t]{2}{*}{ Fundament al I } & 19 & 19 & 38 & \\
\hline & $50 \%$ & $50 \%$ & $100 \%$ & \\
\hline \multirow[t]{2}{*}{ Fundamental II } & 1 & 0 & 1 & \\
\hline & $100 \%$ & $0 \%$ & $100 \%$ & \\
\hline \multirow[t]{2}{*}{ Fundamental I Incompleto } & 5 & 6 & 11 & \\
\hline & $45 \%$ & $55 \%$ & $100 \%$ & \\
\hline \multirow[t]{2}{*}{ Médio } & 2 & 3 & 5 & \\
\hline & $40 \%$ & $60 \%$ & $100 \%$ & \\
\hline Total & 35 & 35 & 70 & \\
\hline
\end{tabular}


A tabela 2 mostra a comparação entre a idade, IMC e perfil glicêmico dos PAF e NPAF. Nos
PAF observa-se uma diminuição do IMC e do perfil glicêmico quando comparados aos NPAF.

Tabela 2 - Comparação entre idade, IMC e perfil glicêmico dos PAF e NPAF. Fortaleza, CE. 2008.

\begin{tabular}{cllllll}
\hline Prática de Atividade Física & Variável & n & Média & DP & Mínimo & Máximo \\
\hline \multirow{2}{*}{ Não } & & & & & & \\
& Idade & 35 & 67,40 & 5,63 & 60,00 & 82,00 \\
& IMC & 35 & 29,38 & 4,11 & 20,40 & 38,70 \\
& Perfil Glicêmico & 35 & 9,47 & 2,47 & 4,90 & 14,00 \\
& & & & & & \\
\multirow{2}{*}{ Sim } & 35 & 67,94 & 4,98 & 60,00 & 77,00 \\
& Idade & 35 & 27,68 & 4,06 & 20,00 & 38,50 \\
& IMC & 35 & 9,39 & 2,36 & 5,50 & 13,50 \\
\hline
\end{tabular}

$\mathrm{Na}$ tabela 3, observa-se o tipo, que os idosos diabéticos praticavam alguma frequência, duração (min) e o tempo (meses) atividade física.

Tabela 3 - Tipo, frequência, duração e tempo que os idosos diabéticos praticam atividade física. Fortaleza, CE. 2008.

\begin{tabular}{lc}
\hline & $\begin{array}{c}\text { Diabéticos PAF } \\
(\mathrm{n}=35) \\
\mathrm{N}(\%)\end{array}$ \\
\hline Tipo de Ativida de Física & \\
Caminhada & $18(51,5)$ \\
Alongamento & $9(25,7)$ \\
Hidroginástica & $5(14,3)$ \\
Musculação & $2(5,7)$ \\
Ciclismo & $1(2,8)$ \\
Frequência por semana (vezes /semana) & \\
3 x / semana & $23(65,7)$ \\
5 x /sema na & $10(28,5)$ \\
6 x/semana & $2(5,8)$ \\
Duração da sessão diária (minutos) & \\
15 a 30 & $28(80,0)$ \\
40 a 60 & $7(20,0)$ \\
Tempo de pratica (meses) & \\
1 a 10 & $24(68,5)$ \\
11 a 20 & $6(17,2)$ \\
mais de 20 & $5(14,3)$ \\
\hline
\end{tabular}


A caminhada foi a atividade física mais realizada pelos idosos $(51,5 \%)$, a maioria dos idosos realiza atividade física pelo menos três vezes na semana $(65,7 \%)$, a sessão de exercícios diária durava 15 a 30 minutos (80\%) e o tempo de prática foi de 1 a 10 meses (68,5\%).
Em relação à média do IMC dos PAF e NPAF, observamos que aqueles que praticavam atividade física apresentaram uma diminuição do IMC, se comparados àqueles que não faziam nenhuma atividade física (tabela 4).

Tabela 4 - Comparação de médias de IMC entre os PAF e NPAF. Fortaleza, CE. 2008.

\begin{tabular}{ccccc}
\hline Comparação & Diferença & p-valor & \multicolumn{2}{c}{ IC95\% } \\
\hline Não-Sim & -1.70 & 0.09 & -3.65 & 0.25 \\
\hline
\end{tabular}

A tabela 5 mostra a comparação das médias do perfil glicêmico entre os PAF e NPAF; notou- se que os PAF apresentaram um perfil glicêmico mais baixo.

Tabela 5 - Comparação de médias do perfil glicêmico entre os PAF e NPAF.

\begin{tabular}{ccccc}
\hline Comparação & Diferença & p-valor & \multicolumn{2}{c}{ IC95\% } \\
\hline Não-Sim & 0,09 & 0,88 & $-1,07$ & 1,24 \\
\hline
\end{tabular}

\section{DISCUSSÃO}

No presente estudo, observou-se um número maior de participantes do sexo feminino, o que comprova o fenômeno de feminização do processo do envelhecimento, conforme apontam Freire \& Tavares..$^{21} \mathrm{~A}$ feminização vem ocorrendo desde o final da década de 80 , mas há muito tempo tem sido superior, no país, o número absoluto de mulheres idosas, quando comparado ao de homens idosos. Segundo dados da última Pesquisa Nacional de Amostra por Domicílios, entre os idosos o percentual feminino vem aumentando lentamente: em 1981, com 52,6\%; em 1999, com $55,3 \%$; e em 2004 , com $56,1 \%$ de mulheres idosas. ${ }^{22}$

Sobre as variáveis antropométricas, verificamos que os idosos DM2 que PAF apresentaram sobrepeso e perfil glicêmico acima do normal. Mas quando comparados aos NPAF, encontravam-se em melhor condição.

O excesso de peso atinge cerca de um terço da população adulta e tem apresentado tendência crescente nas últimas décadas, mesmo entre as pessoas idosas. ${ }^{23}$ Estima-se que entre $80 \%$ e $90 \%$ dos indivíduos acometidos pelo DM tipo 2 são obesos ou estão acima do peso..$^{24,25}$

Nesta pesquisa se observou, pela avaliação antropométrica, que os pacientes apresentavam excesso de peso, confirmando dados de outros estudos que revelam que a obesidade e/ou sobrepeso estão presentes na maioria dos pacientes diabéticos tipo $2 .^{26,27}$

É importante salientar que a permanência de níveis glicêmicos elevados relaciona-se com sérias complicações crônicas de alta prevalência em pacientes DM2.

Estudo realizado por Van et al. ${ }^{28}$ identificou a associação entre inadequados perfis lipídicos e níveis glicêmicos de jejum mais elevados com a presença de excesso de peso e/ou adiposidade central, evidenciando a necessidade de intervenção imediata em pacientes DM2 com excesso de peso ou adiposidade central para melhor controle 
metabólico da doença e menor risco de complicações crônicas futuras.

Um estudo dinamarquês demonstrou que, nos anos de 1996 e 1997, a prevalência de diabetes foi de $12,3 \%$ em homens e $6,8 \%$ em mulheres com 60 ou mais anos de idade. Comparando dados da mesma população obtidos em 1974 e 1975, o aumento do número de casos foi de $58 \%$ para os homens e $21 \%$ para as mulheres. Um concomitante aumento no IMC, no mesmo período, explicaria o aumento na prevalência de diabetes. ${ }^{29}$ É interessante observar a estimativa de que $30 \%$ a $60 \%$ dos indivíduos com esta doença, na comunidade, estejam sem o diagnóstico. ${ }^{26}$

Em relação à prática de atividade física, observamos que aqueles que a praticavam regularmente apresentavam um nível de glicêmico melhor do que os idosos que não praticam nenhuma atividade física.

Tideiksaar $^{30}$ apresenta resultados de uma metaanálise ao citar inúmeras pesquisas internacionais, mostrando a importância de programas de condicionamento físico e de reabilitação, tanto para idosos que vivem na comunidade quanto para aqueles institucionalizados. Os resultados mostram a eficácia desses programas em melhorar a capacidade funcional, a mobilidade e a independência dos idosos.

A atividade física regular vem sendo considerada uma forma de manutenção da aptidão física em indivíduos idosos, citada na literatura como um modo de atenuar e reverter a perda de massa muscular, contribuindo para preservar a autonomia funcional e o envelhecimento saudável. ${ }^{31}$

Estudos como o de Helmrich, Ragland, Leung, Paffenbarger ${ }^{32}$ têm mostrado que a prática de atividade física por pacientes com DM2 é importante para o aumento da sensibilidade à insulina. Afirmam ainda que a prática de atividade física pode prevenir o aparecimento desta doença em pessoas que tenham um risco elevado para contraí-la.

\section{CONCLUSÃO}

A partir dos resultados obtidos na análise dos dados, foi possível mostrar os benefícios que a atividade física proporciona aos indivíduos no processo de envelhecimento.

$\mathrm{Na}$ análise dos resultados obtidos, podemos concluir que a atividade física proporcionou uma diminuição nas variáveis antropométricas e no perfil glicêmico dos idosos diabéticos praticantes de atividade física regular.

A atividade física é fundamental para minimizar o desenvolvimento precoce de doenças crônicas, possibilitando uma longevidade com maior qualidade de vida. 


\section{REFERÊNCIAS}

1. Chaimowicz F. A saúde dos idosos brasileiros às vésperas do século XXI: problemas, projeções e alternativas. Rev Saúde Publica 1997; 31(1): $184-$ 202.

2. Menezes TN, Marucci MFN. Antropometria de idosos residentes em instituições geriátricas, Fortaleza,CE. Rev Saúde Pública 2005; 39(2).

3. Campos MTFS, Monteiro JBR, Ornelas APRC. Fatores que afetam o consumo alimentar e a nutrição do idoso. Rev. Nutr, Campinas 2000; 13(3)

4. Campos MAG; e colaboradores. Estado nutricional e fatores associados em idosos. Rev. Assoc. Méd. Brás 2006; 52(4)

5. Veras RP, Ramos LR, Kalache A. Crescimento da população idosa no Brasil: transformações e conseqüências na sociedade. Rev Saúde Pública 1987 jun; 21(3).

6. Alves MI, Veras RP. A população idosa no Brasil: considerações acerca do uso de indicadores de saúde. In: Minayo MCS. Os muito Brasis: saúde e população na década de 80. 1 ed. São Paulo: HUCITEC,ABRASCO; 1995. p $321-7$.

7. Costa EFA, Monego ET. Avaliação geriátrica ampla. Rev. Da UFG 2003 dez; 5(2). Disponível em: http://www.proec.ufg.br

8. Matsudo SM, Matsudo VKR, Barros Neto TL. Impacto do envelhecimento nas variáveis antropométricas, neuromotoras e metabólicas da aptidão física. Rev. Brás. Ciênc e Mov. 2000 abril; 8(4): 21-32.

9. Ong KL, Cheung BM, Man YB, Lau CP, Lam KS. Prevalence, awareness, treatment, and control of hypertension among United States adults 1999-2004. Hypertension 2007; 49: 69-75.

10. Sakharova OV, Inzucchi SE. Treatment of diabetes in the elderly. Addressing its complexities in this high-risk group. Postgrad Med 2005; 9(118): 19-26.

11. Knowler WC, Barrett-Connor E, Fowler SE, Hamman RF, Lachin JM, Walker EA, et al. Reduction in the incidence of type 2 diabetes with lifestyle intervention or metformin. $\mathrm{N}$ Engl J Med 2002; 346: 393-403.

12. Nguyen HQ, Ackermann RT, Berke EM, Cheadle A, Williams B, Lin E, et al. Impact of a managed-medicare physical activity benefit on health care utilization and costs in older adults with diabetes. Diabetes Care. 2007; 30: 43-8.
13. Goff DC, Jr., Bertoni AG, Kramer H, Bonds D, Blumenthal RS, Tsai MY, et al. Dyslipidemia prevalence, treatment, and control in the MultiEthnic Study of Atherosclerosis (MESA): gender, ethnicity, and coronary artery calcium. Circulation 2006; 113: 647-56.

14. Passos VM, Barreto SM, Diniz LM, Lima-Costa MF. Type 2 diabetes: prevalence and associated factors in a Brazilian community-the Bambui health and aging study. Sao Paulo Med J. 2005; 123: 66-71.

15. Marques AP, Arruda IK, Espirito Santo AC, Raposo MC, Guerra MD, Sales TF. [Prevalence of obesity and associated factors in elderly women]. Arq Bras Endocrinol Metabol 2005; 49: 441-8.

16. Pearlman BL. The new cholesterol guidelines. Applying them in clinical practice. Postgrad Med. 2002; 112: 13-6, 9-22, 5-6 passim.

17. Sociedade Brasileira de Diabetes. Tratamento e acompanhamento do Diabetes Mellitus. Rio de Janeiro: Diagraphic; 2007. p.168.

18. SAS Institute Inc., SAS/STAT® User's Guide, Version 9, Cary, NC: SAS Institute Inc., 2003.

19. Conselho Nacional de Saúde (Brasil). Normas de pesquisa envolvendo seres humanos. Res. CNS 196/96. Bioética 1996; 4 Suppl:15-25.

20. Coelho Filho JM, Ramos LR. Epidemiologia do envelhecimento no Nordeste do Brasil: resultados de inquérito domiciliar. Rev Saúde Pública 1999; 33(5): 445-53.

21. Freire Jr RC, Tavares MFL. A promoção de Saúde nas instituições de longa permanência: uma reflexão sobre o processo de envelhecimento no Brasil. Revista brasileira de geriatria e gerontologia 2006; 9(1): 83-92.

22. Fundação Instituto Brasileiro de Geografia e Estatística (2004). Pesquisa Nacional de Amostra por Domicílio no Brasil (PNAD) [acesso 2006 jul 20]. Disponível em URL: http:// www.ibge.gov.br.

23. Bós AMG, Bós AJG. Determinants of elders choice between private and public health care providers. Rev. Saúde Pública. 2004 [capturado 2007 Nov 6 ]; Disponível em: http:/ / www.scielo.br/scielo.php?pid=S0034$89102004000100016 \&$ script $=$ sci_arttext\&tlng=pt\#back1

24. World Health Organization. Physical status: the use and interpretation of anthropometry. Geneva: World Health Organization; 1995 Contract No.: Document Number.

25. Spirduso W. Dimensões físicas do envelhecimento. São Paulo: Manole; 2005. 
26. Voss L. The measuremet of human growth: a historical review. In: Dasgupta P, Hauspie R, editors. Perspectives in human growth development and maturation. Springer; 2001. p. 3-16.

27. Lima-Costa MF, Veras R. Aging and public health. Cad Saude Publica. 2003 May-Jun; 19(3): 701, 0.

28. Porto F. Avaliação Postural dos Idosos de Porto Alegre-Rs com o Uso da Técnica de Moiré de Sombra. [Tese]. In press 2008.

29. Rana JS, Li TY, Manson JE, Hu FB. Adiposity compared with physical inactivity and risk of type 2 diabetes in women. Diabetes Care 2007; 30: 53-8.
30. Tideiksaar R. As quedas na velhice: prevenção e cuidados. São Paulo: Organização Andrei Editora LTDA; 2003.

31. Fabrício SCC,Rodrigues RAP, Costa JR. Causas e conseqüências de quedas de idosos atendidos em hospital público. Rev Saúde Pública 2004; 38: 93-99.

32. Helmrich SP, Ragland DR, Leung RW, Paffenbarger RS. Physical activity and reduced occurrence of non-insulin-dependent diabetes mellitus. New England Journal Medical 1999; 325: $147-52$.

Recebido: 25/11/2008

Revisado: 24/10/2009

Aprovado: 23/11/2009 
